\title{
Estimation of relatedness among non-pedigreed Yakutian cryo-bank bulls using molecular data: implications for conservation and breed management
}

\author{
IIma Tapio', Miika Tapio', Meng-Hua Li1, Ruslan Popov², Zoya Ivanova² and Juha Kantanen*1
}

\begin{abstract}
Background: Yakutian cattle, the last remaining native cattle breed in Siberia, are well adapted to the extreme subarctic conditions. Nowadays only ca. 1200 purebred animals are left in Yakutia. The semen of six Yakutian bulls was stored in a cryo-bank without any pedigree documentation because of the traditional free herding style of the population.

Methods: To clarify the genetic relatedness between these bulls and to provide recommendations to use their semen in future conservation and breed management programs, we have analysed 30 autosomal microsatellites and mitochondrial DNA sequences in 60 individuals including the six for which semen has been stored. Four relatedness estimators were calculated. In addition, we assessed the value of the cryo-bank bulls for the preservation of genetic variation of the contemporary Yakutian cattle by calculating allelic and gene diversity estimates and mean molecular coancestries.

Results: On the basis of microsatellite variability, including the Yakutian cryo-bank bulls increases the allelic variation in the contemporary population by $3 \%$ and in the male subpopulation by $13 \%$. In terms of the mean molecular coancestries, they are less related to the contemporary cow population than the breeding bulls and therefore could be used to reduce inbreeding in the living population. Although 30 loci are insufficient to resolve definitely their relatedness categories, the data suggest four pairs of cryo-bank bulls as possible half-sibs.

Conclusions: Our results show that even relatively limited cryo-bank storage of semen can carry allelic variation through a bottleneck. We propose a breeding scheme based on the rotation of breeding females and the division of cryo-bank bulls into three groups. Thus, if molecular data (e.g. autosomal microsatellite genotypes) for the contemporary population are available and based on relatively small-scale laboratory analyses, it is possible to avoid serious mistakes in their use for breeding applications. The approach suggested here based on the use of Yakutian cryo-bank semen can be easily extended to cryo-bank materials of other animals in future breeding programs.
\end{abstract}

\section{Background}

Yakutian cattle are the last remaining native cattle breed of the East Asian 'Turano-Mongolian' type of Bos taurus in Siberia. They are distributed in the north-eastern region of the Sakha Republic (Yakutia) of the Russian Federation [1-3]. These cattle possess a number of traits, such as solid trunk, short strong legs and long thick win-

* Correspondence: juha.kantanen@mtt.fi

1 Biotechnology and Food Research, MTT Agrifood Research Finland, Jokioinen, Fl-31600 Finland

Full list of author information is available at the end of the article ter coat, which make them adapted to the extreme subarctic conditions. Moreover, efficient thermoregulation, quick formation of subcutaneous fatty tissue and low metabolic rates at low temperatures (even down to $-60^{\circ} \mathrm{C}$ ) allow them to survive in harsh environments under poor feed conditions (e.g. [3]). Ancestors of Yakutian cattle can be traced back to indigenous cattle in Siberia, which migrated with the Yakuts $c a$. 1,000 years ago from the southern Baikal region to the northern regions of the Lena and Yana rivers. Yakutian cattle were purebred until 
1929 and, from then on, were subjected to extensive crossbreeding with productive breeds [2]. Consequently, only ca. 1200 purebred Yakutian cattle individuals remain in three villages in the district of Eveno-Bytantaisky, one village of Uluu-Syhyy and four different farms close to Yakutsk City [1]. Currently the population comprises only 525 breeding cows and 28 breeding bulls. Yakutian cattle are classified as an endangered breed by the Food and Agriculture Organization of the United Nations (FAO) [4]. However, recent studies in a continental context have suggested that this breed is highly interesting for the conservation of cattle genetic diversity [3,5]. There is a need to conserve the breed for future cattle breeding actions as well as for scientific and cultural purposes.

Maintaining genetic variability and avoiding inbreeding are of great importance in the management of small animal populations. Inbreeding has a negative effect on fitness, productivity and several other phenotypic traits [6]. Meanwhile, a reduction in gene and allele diversity might reduce a population's response to environmental changes or artificial selection in the future $[7,8]$. Thus, ex situ banking of embryos, oocytes and semen plays a fundamental role in the conservation and management of small farm animal breeds [9]. Storage of genetic material represents a reservoir of a breed's genetic diversity and could be used to re-establish a breed, if needed. The only genetic material stored ex situ for Yakutian cattle is the semen from six bulls collected between 1980 and 1986. However, because of the traditional free herding style of these cattle in summer pastures, where several bulls mate randomly within a herd, pedigree records of these six bulls are not available and, thus, the traditional pedigreebased control of inbreeding is impossible in a meaningful way.

In the absence of pedigree records, molecular data from autosomal, maternally inherited mitochondrial DNA (mtDNA) or from paternally inherited Y-chromosomal markers can be used to estimate relatedness between animals [10-12]. The widely applied statistical approaches to infer relatedness among individuals can be classified into two categories: one involves the explicit pedigree reconstruction among all individuals in the sample; and the other is based on the best pairwise relationship between two individuals at a time based on either relatedness estimation [13-15] or likelihood techniques [16,17]. The weakness of the pairwise methods is that they do not take into account information from the reference population and the difficulty in distinguishing among relationships with similar patterns of alleles (e.g. [18]). However, pedigree reconstruction methods have been applied mainly to the reconstruction of full-sib families [19].

Survival of the last native cattle breed in Siberia, Yakutian cattle, is important for the local human community as a source of food and income [1], but also because it presents extreme adaptive potentials of the cattle species in general. However, due to the small census size, Yakutian cattle require a careful management strategy. Longterm cryo-conservation of embryos and semen should be considered seriously as they represent a resource for ongoing breeding activities and a secure way of preserving genetic diversity within the breed, should the living population encounter problems. Although molecular measures of genetic relatedness do not necessarily agree exactly with the true relatedness coefficients calculated from the pedigree records (but see [20]), they are the best relatedness indicators in the absence of recorded pedigree information (e.g. [11]). Therefore, the specific goals of the current study were to estimate genetic relatedness among the six Yakutian cryo-bank bulls using pairwise and pedigree reconstruction methods based on the analysis of autosomal microsatellites and mtDNA sequences. We have also assessed how much genetic variation such a limited ex situ bank could add to the contemporary population of Yakutian cattle. Our aim was to solve a practical conservation problem in a highly valued cattle breed and to see how helpful basic population genetics analyses are in solving such a breed management question. Our results also provide recommendations for future conservation and use of the six cryo-bank semen.

\section{Methods}

\section{Sampling and data extraction}

Genomic DNA was extracted from the frozen semen samples of six Yakutian cattle cryo-bank bulls (named Keskil, Moxsogol, Radzu, Erel, Sarial and Alii), whose semen had been stored for more than 20 years, according to the method described by [21]. For the genetic diversity comparison, a reference population consisting of 54 randomly sampled Yakutian cattle individuals from the State farm in the village of Kustur (17 individuals) and from private farms in the villages of Batagai-Alyta (17), Kustur (4) and Uluu-Syhyy (16) in the Sakha Republic were also included in the analysis [3]. The reference population included samples of 37 cows and 17 bulls, referred hereafter to as 'the cow subpopulation' and 'the bull subpopulation', respectively. Genotypes of the reference population using the same set of 30 autosomal microsatellites were obtained from a previous study by Li et al. [3] .

\section{Molecular analysis}

To determine the levels of mtDNA variability, DNA samples of the six Yakutian cryo-bank bulls were sequenced for a 375-nucleotide fragment of the mtDNA control region using the primers published in [22]. The sequenced fragment covers bases 15,960 to 16,334 as compared to the complete cattle mtDNA sequence (NC006853). Standard double-stranded sequencing was performed with DYEnamic ET Terminator Kit (Amer- 
sham Biosciences) using the primers for polymerase chain reaction (PCR) and $10 \mu \mathrm{L}$ of purified PCR-product on a MegaBACE 500 DNA Sequencer (Amersham Biosciences). Complementary sequences were combined using the SEQUENCHER v4.6 software (Gene Codes Co, Ann Arbor, MI, USA). In addition, sequences of 24 random individuals from the reference population (accession numbers FJ014247-FJ014270) were obtained from a recent study [23]. The six Yakutian cryo-bank bulls and international reference animals were genotyped for the same set of 30 microsatellites (Table 1) as described in [3]. Information on primers and PCR conditions can be found in the Cattle Diversity Database http://www.projects.roslin.ac.uk/cdiv/markers.html.

\section{Statistical analysis}

To characterise the maternal lineages, multiple alignments of mtDNA sequences were performed using ClustalX version 1.81 [24]. The size of the aligned mtDNA control region fragment was 255 nucleotides between bases 16,021 and 16,275 compared to the complete cattle mtDNA sequence (NC006853). The number of haplotypes was estimated and pairwise genetic distances between haplotypes were calculated based on the number of nucleotide differences using MEGA version 3.1 [25].

Genetic variability of the autosomal microsatellite loci in the whole Yakutian cattle sample (60 individuals) was quantified by the observed number of alleles $\left(A_{\mathrm{O}}\right)$ and polymorphism information content (PIC) per locus using the program Microsatellite TOOLKIT [26]. Locus-wise tests for Hardy-Weinberg equilibrium (HWE) due to heterozygote deficiency were performed with 10,000 Monte Carlo randomisations [27] and the 'U' statistic test [28] as implemented in the programs GENEPOP version 4.0 [29] and ML-Relate [17], respectively. The program GENEPOP was also used in the Fisher's exact tests for genotypic linkage disequilibrium (LD) between all pairs of microsatellites with a Markov chain method of 50,000 iterations and 100 batches.

Relationships among the six Yakutian cryo-bank bulls were estimated with the pairwise relatedness estimators, $r_{\mathrm{W}}[15]$ and $r_{\mathrm{QG}}$ [13], using the program SPAGeDi version 1.2 [30]. The calculation was based on autosomal microsatellite genotypes in all 60 individuals. Furthermore, pairwise relationships between the bulls were calculated with the maximum-likelihood estimator $r_{\mathrm{K}}$ using the program ML-Relate [17]. Performances of $r_{\mathrm{W}}, r_{\mathrm{QG}}$, and $r_{\mathrm{K}}$ were evaluated using a simulation approach as implemented in PEDAGOG [31]. Allele frequencies of the 30 microsatellites obtained from all 60 individuals were used as input data. Distribution of pairwise relatedness $(R)$ estimates for each of the four simulated relationship categories [unrelated (UR), half-sibs (HS), full-sibs (FS), and parent-offspring $(\mathrm{PO})]$ was based on the simulated geno-
Table 1: Information on microsatellite markers

\begin{tabular}{|c|c|c|c|c|}
\hline Locus & BTA & $A_{\mathrm{O}}$ & PIC & $P$ \\
\hline BM1824 & 1 & 4 & 0.39 & 0.547 \\
\hline BM2113 & 2 & 6 & 0.58 & 0.834 \\
\hline ETH10 & 5 & 5 & 0.39 & 0.543 \\
\hline ETH225 & 9 & 5 & 0.63 & 0.355 \\
\hline ETH3 & 19 & 4 & 0.60 & 0.908 \\
\hline HEL5 & 21 & 5 & 0.62 & 0.097 \\
\hline ILSTS005 & 10 & 3 & 0.32 & 0.813 \\
\hline INRA023 & 3 & 5 & 0.52 & 0.879 \\
\hline INRA035 & 16 & 3 & 0.18 & 0.002 \\
\hline INRA005 & 12 & 4 & 0.61 & 0.652 \\
\hline INRA063 & 18 & 3 & 0.33 & 0.407 \\
\hline BM1818 & 23 & 2 & 0.33 & 0.089 \\
\hline CSSM66 & 14 & 8 & 0.65 & 1.000 \\
\hline ETH152 & 5 & 4 & 0.51 & 0.969 \\
\hline HEL1 & 15 & 5 & 0.57 & 0.200 \\
\hline HEL13 & 11 & 4 & 0.44 & 0.143 \\
\hline HEL9 & 8 & 6 & 0.62 & 0.056 \\
\hline ILSTS006 & 7 & 6 & 0.67 & 0.115 \\
\hline INRA032 & 11 & 3 & 0.45 & 0.103 \\
\hline INRA037 & 11 & 5 & 0.52 & 0.265 \\
\hline TGLA227 & 18 & 8 & 0.68 & 0.421 \\
\hline TGLA126 & 20 & 4 & 0.68 & 0.216 \\
\hline TGLA122 & 21 & 6 & 0.67 & 0.745 \\
\hline HAUT24 & 22 & 4 & 0.50 & 0.747 \\
\hline HAUT27 & 26 & 8 & 0.69 & 0.389 \\
\hline CSRM60 & 10 & 7 & 0.62 & 0.206 \\
\hline MM12 & 9 & 4 & 0.51 & 0.181 \\
\hline ETH185 & 17 & 5 & 0.61 & 0.328 \\
\hline TGLA53 & 16 & 10 & 0.67 & 0.134 \\
\hline SPS115 & 15 & 4 & 0.41 & 0.264 \\
\hline Mean & - & 5 & 0.53 & - \\
\hline
\end{tabular}

For each locus are given the chromosomal location (BTA), and the summary statistics per locus such as the number of observed alleles $\left(A_{\mathrm{O}}\right)$, polymorphism information content (PIC) and $P$-values for the deviation from Hardy-Weinberg equilibrium across the total 60 Yakutian samples

types from 1000 individual-pairs each. The sampling variance was calculated as the standard deviation of the mean $R$ estimate for each simulation category separately. The bias among estimators was tested by comparing the mean and the expected $R$ values (UR 0.0; HS 0.25; FS and PO 0.5). Two-tailed $t$-tests were used to evaluate the significance of potential bias. Critical significance values 
were adjusted for multiple tests with sequential Bonferroni correction.

Pedigree reconstruction among all individuals in the sample was performed using PARENTAGE version 1.0 [32]. Two chains with burn-in of 200 iterations, thinning of 400 and 2000 samples were applied. A Dirichlet prior for the allele frequencies was used and the prior for the distribution of offspring between males and females was set to be gamma $(1,2)$. Influence of the six Yakutian cryobank bulls on the genetic variability of the reference population and the bull subpopulation were investigated by calculating basic statistics such as gene and allelic diversities.

Molecular coancestry is similar to the genealogical coancestry coefficient [33] but is defined as the probability that two alleles taken at random, one from each individual, are identical by state. To test if the six Yakutian cryo-bank bulls were less related to the cow subpopulation (37 cows) than the bull subpopulation (17 bulls), we used the program MOL_COANC version 1.0 [34] to calculate the mean molecular coancestry for the whole Yakutian cattle population (60 individuals), for the all the bulls (23 bulls comprising the six cryo-bank bulls and the 17 reference bulls), for the 17 reference bulls, for six Yakutian cryo-bank bulls and for each of the 23 bulls separately. Mean molecular coancestry [33] between each bull and every cow was also calculated. The difference between the bull subpopulation (17 reference bulls) and the group of six Yakutian cryo-bank bulls was tested using a two-sample permutation test by the Hothorn and Hornik exactRankTests version 0.8-12 package for the $R$ language.

\section{Results}

\section{Mitochondrial data}

Screening of the $255 \mathrm{nt}$ fragment of the mtDNA control region identified 11 haplotypes defined by 17 variable sites that belong to the taurine mtDNA sub-haplogroups T2, T3 and T4 (Additional file 1) [35,36]. Six haplotypes were individual-specific, three haplotypes were shared by two samples, one haplotype was shared by four samples and the most common haplotype was shared by 14 individuals. MtDNA sequences of the six Yakutian cryo-bank bulls (accession numbers FJ014464-FJ014469) were characterized by six different haplotypes, four of which were not observed in the contemporary samples (Additional file 1). The average number of pairwise nucleotide differences among all 11 haplotypes was 3.78, ranging from 1 to 8 among pairs of comparison. The number of pairwise nucleotide differences among the six haplotypes observed in the six Yakutian cryo-bank bulls varied from 2 to 7 with an average number of 4.53. We did not find any mtDNA haplotype shared by all six Yakutian cryo-bank bulls, which indicates that these bulls cannot be full-sibs or maternal half-sibs.

\section{Microsatellites and relatedness}

One hundred and fifty alleles were detected in the 60 Yakutian cattle individuals across the 30 microsatellites. The number of observed alleles varied from 2 to 10 per locus (Table 1). The average PIC across the loci for the complete sample was 0.532 , with the lowest PIC observed at INRA035 (0.176) and the highest at HAUT27 (0.685). No significant $(P<0.05)$ deviations from LE were observed in the pairwise microsatellite comparisons after sequential Bonferroni correction was applied. Significant $(P<0.05)$ heterozygote deficiency was detected only at INRA035 (Table 1), which is probably due to the presence of non-amplifying alleles (e.g. [37]). It is also possible that the locus INRA035 is near a gene or within a genomic region under directional selection and this would be interesting to investigate further.

We calculated pairwise relatedness estimates between the six Yakutian cryo-bank bulls with and without the locus INRA035. These calculations of relatedness were further adjusted to accommodate non-amplifying alleles by the option as implemented in the ML-Relate program. Neither the exclusion of the locus nor the inclusion of the non-amplifying alleles had a significant effect on the relatedness estimates (not shown). Therefore, the results presented in the study are based on the full set of 30 microsatellites (Additional file 2).

Mean $r_{\mathrm{W}}$ and $r_{\mathrm{QG}}$ estimates and their standard deviations calculated for four simulated relatedness distributions are presented in Additional file 3. Performances of both pairwise relatedness estimators were similar to each other with only minor differences in variance estimates. $r_{\mathrm{QG}}$ had a slightly smaller (by 0.004) sampling variance for the distribution of UR individuals, while $r_{\mathrm{W}}$ performed better in the remaining categories (HS by 0.002 , FS by 0.01 and $\mathrm{PO}$ by 0.015$)$. In three out of eight cases, mean $R$ deviated significantly from the expected value $(P<0.013$ after sequential Bonferroni correction). The bias for $r_{\mathrm{W}}$ for $\mathrm{UN}$ pairs was downwards, while that for $r_{\mathrm{W}}$ and $r_{\mathrm{QG}}$ in the category of PO was upwards (Additional file 3 ). The performance of $r_{\mathrm{K}}$ was very similar to that of $r_{\mathrm{W}}$ (results not shown) apart from negative values being converted to zero relatedness.

Ten out of 15 pairwise $R$-estimates between the six Yakutian cryo-bank bulls approached zero or fell below it. The remaining five bull-pairs exhibited $R$-values ranging from 0.124 to 0.276 for $r_{\mathrm{W}}$ and from 0.180 to 0.295 for $r_{\mathrm{QG}}$ (Additional file 2). All pairwise $R$ values were plotted on the distribution of four simulated relatedness categories (Figure 1). When the $r_{\mathrm{W}}$ estimator was used, one pair 


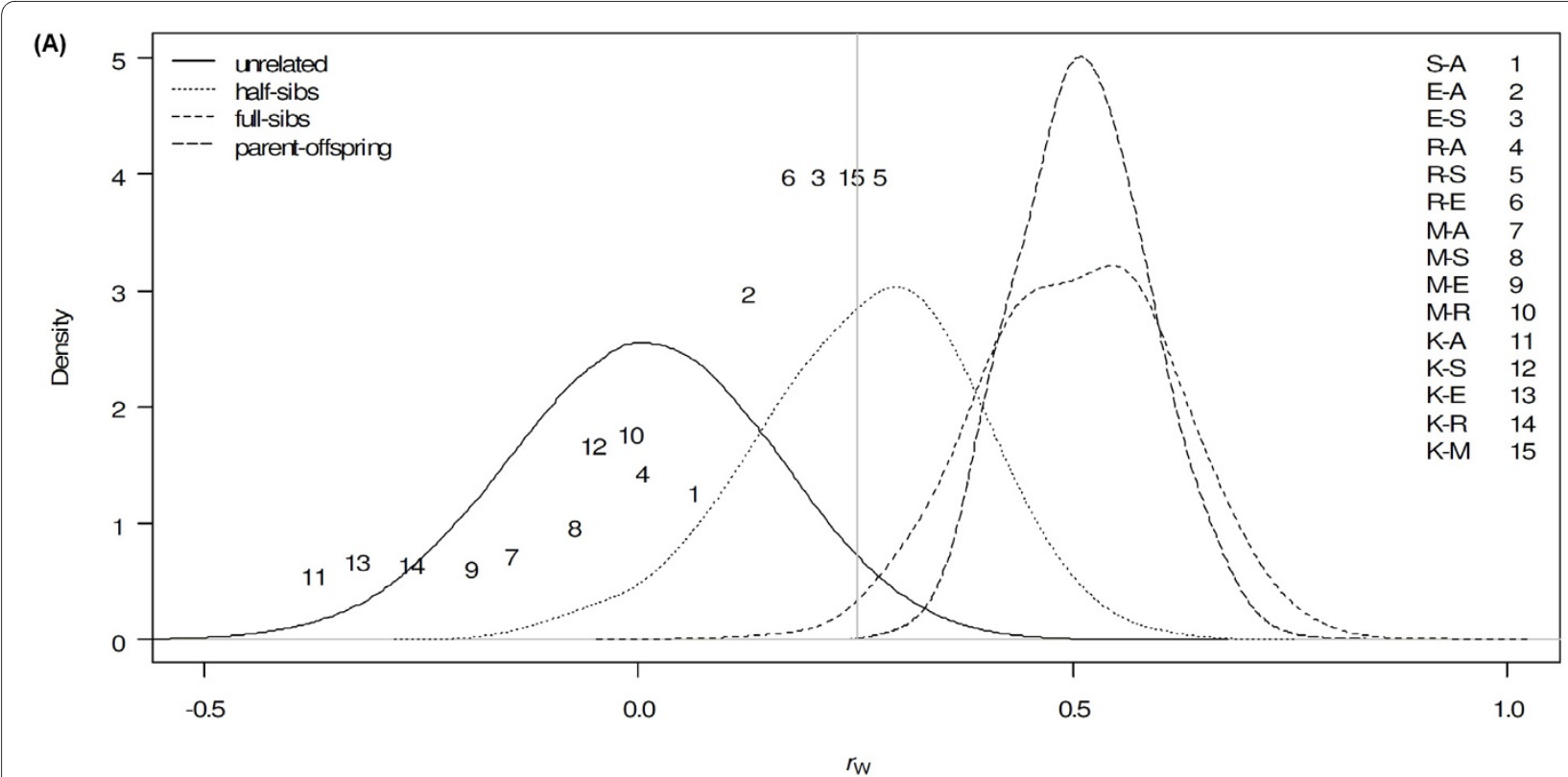

(B)

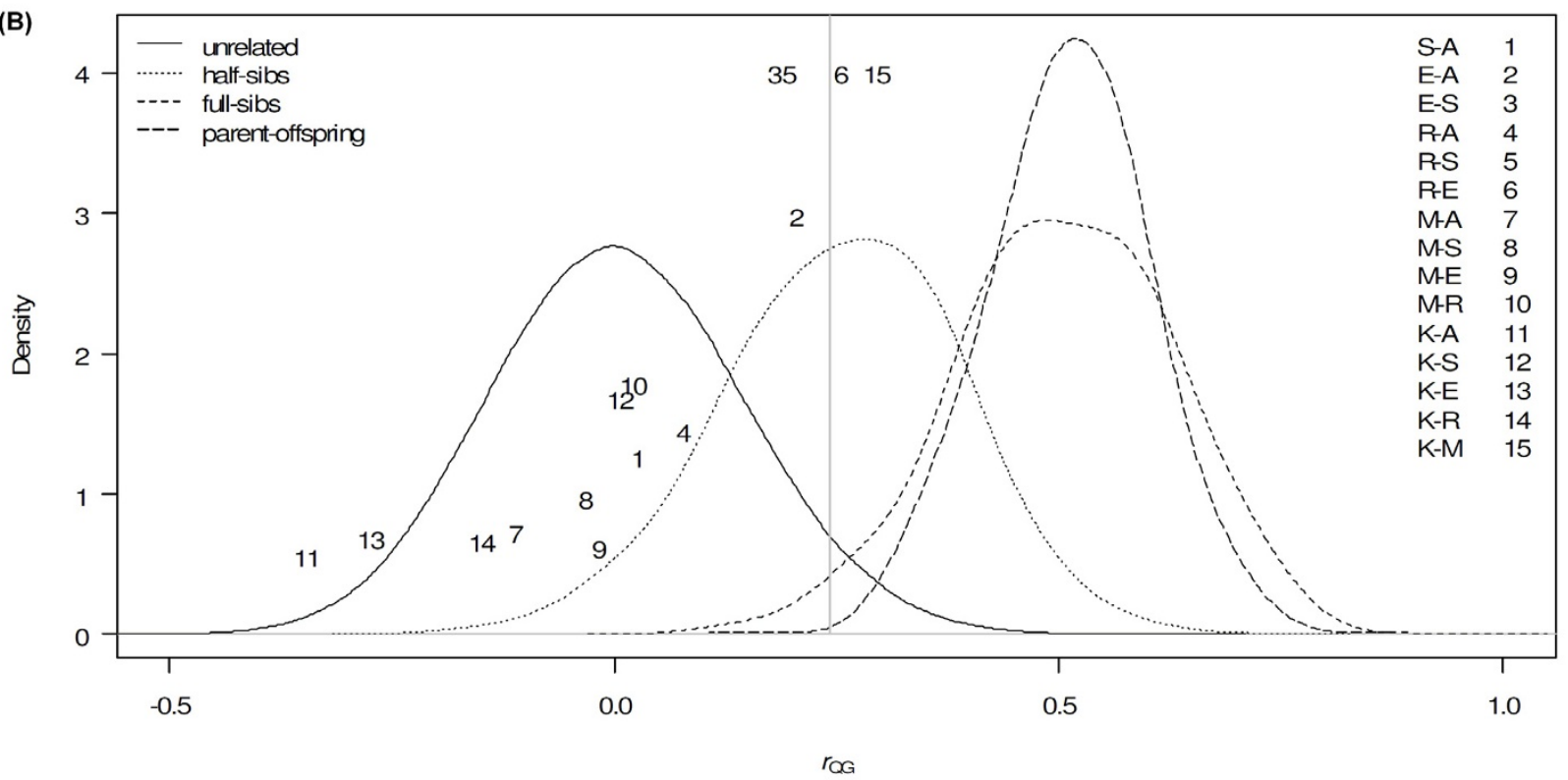

Figure 1 Pairwise relatedness of Yakutian cryo-bank bulls. Values are calculated using (A) $r_{\mathrm{W}}$ and $(\mathbf{B}) r_{\mathrm{QG}}$ relatedness estimators plotted on a distribution of four simulated relationship categories: unrelated, half-sibs, full-sibs and parent-offspring; the vertical line represents the $95^{\text {th }}$ percentile for simulated unrelated individuals; the position of pairwise values in regards to the $Y$-axis was designed based on the estimates from the $r_{K}$ relatedness estimator and was calculated as 3 divided by the cases when the log likelihood of $R$ for the second closest relationship is smaller than the most likely relationship; abbreviations for Yakutian cryo-bank bulls are: K-Keskil, M-Moxsogol, R-Radzu, E-Erel, S-Sarial, A-Alii. Y-axis denotes the distribution of posterior probability density based on the simulations of the four relationship categories using the two relatedness estimators $r_{\mathrm{W}}$ and $r_{\mathrm{QG}}$, respectively.

(Radzu:Sarial, $R=0.276$ ) fell outside the 95\% confidence interval for simulated UR individuals (the $95^{\text {th }}$ upper quantile $=0.252$ ) and was considered to be related (Figure 1a). Two other pairs were identified as related when the $r_{\mathrm{QG}}$ estimator was applied (Keskil:Moxsogol, $R=0.295$; Radzu:Erel, $R=0.255$; the $95^{\text {th }}$ upper quantile $=0.242$ ) (Figure 1b). The ML-Relate program uses simulation to determine which relationships are consistent with genotype data and to compare putative relationships with alternatives. In order to identify possible misclassified individuals, a maximum-likelihood estimator $r_{\mathrm{K}}$ estimated by ML-Relate was applied. Besides the three bullpairs mentioned above, the Erel:Sarial pair $\left(r_{\mathrm{W}}=0.205\right.$; $\left.r_{\mathrm{QG}}=0.180\right)$ had the highest likelihood of being a half-sib 
(Additional file 2). The same four pairs of Yakutian cryobank bulls were also identified as potential half-sibs in the parentage analysis performed using the pedigree reconstruction method among all individuals in the sample (Additional file 4).

\section{Allelic diversity and gene diversity}

Inclusion of the six Yakutian cryo-bank bulls in the calculation increases the within-population genetic variability relative to that in the contemporary reference population (Table 2). For example, the six cryo-bank samples made it possible to add two new alleles at the locus INRA023 and their frequency in the cryo-bank samples is 0.083 . Therefore, compared to the three alleles detected in the 54 contemporary samples from the reference population, a $67 \%$ gain in allelic variation was observed when including the six cryo-bank samples. With the six cryo-bank bulls, the average allelic diversity of the total Yakutian population increased by $3 \%$, while the average allelic diversity of the bulls increased by $13 \%$. Frequencies of alleles specific for the cryo-bank bulls ranged from 0.083 to 0.250 . Three Yakutian cryo-bank bulls, Keskil, Radzu and Alii, carried alleles not detected in the contemporary Yakutian population. Furthermore, all six Yakutian cryo-bank bulls possessed microsatellite alleles that were not found in the contemporary bull subpopulation. The gene diversity would increase by $3.5 \%$ if the six cryo-bank bulls repre- sented the total bull subpopulation in the next generation together with the contemporary cows. The increase in gene diversity would be $1.2 \%$ by adding cryo-bank bulls to the contemporary bull subpopulation in the calculation.

\section{Molecular coancestry}

The mean molecular coancestry was 0.416 for pairwise comparisons among all 60 Yakutian cattle individuals (Table 3). The average molecular coancestry calculated between each Yakutian bull and the cows ranged from 0.344 to 0.465 . Compared with the living contemporary bull subpopulation, the group of six Yakutian cryo-bank bulls showed a significantly lower $(0.395 v s .0 .418$; a permutation test between the two mean values, $P=0.035$ ) mean coancestry with the living contemporary cow subpopulation (Table 3). This indicates that the cryo-bank bulls are good candidates as sires in a breeding program aimed at avoiding inbreeding.

\section{Discussion}

Knowledge on pairwise relatedness is crucial to draft recommendations for further use of cryo-bank bull semen in conservation and breeding programs of domestic animals. In this study, we have estimated pairwise relatedness among the six Yakutian cryo-bank bulls with different estimators based on autosomal and mtDNA genetic variation. Our study has shown that molecular

Table 2: Increase in allelic variation when the six Yakutian cryo-bank bulls are included in the analysis

\begin{tabular}{|c|c|c|c|c|c|c|c|c|}
\hline & \multicolumn{4}{|c|}{ Total $(N=60)$} & \multicolumn{4}{|c|}{ Males $(N=23)$} \\
\hline & $N_{\mathrm{A}}$ & $\%$ & Frequency & Individual & $\mathbf{N}_{\mathrm{A}}$ & $\%$ & Frequency & Individual \\
\hline ETH10 & 0 & 0 & - & & 1 & 25 & 0.167 & Keskil, Erel \\
\hline HEL5 & 0 & 0 & - & & 1 & 25 & 0.083 & Sarial \\
\hline INRA023 & 2 & 67 & 0.083 & Radzu, Alii & 2 & 67 & 0.083 & Radzu, Alii \\
\hline INRA063 & 0 & 0 & - & & 1 & 50 & 0.250 & Keskil, Moxsogol, Sarial \\
\hline CSSM66 & 0 & 0 & - & & 1 & 17 & 0.167 & Erel \\
\hline ILSTS006 & 0 & 0 & - & & 2 & 67 & 0.250 & Keskil, Moxsogol \\
\hline TGLA227 & 1 & 14 & 0.083 & Keskil & 1 & 17 & 0.083 & Keskil \\
\hline TGLA122 & 0 & 0 & - & & 2 & 50 & 0.083 & Moxsogol, Erel \\
\hline HAUT27 & 0 & 0 & - & & 1 & 14 & 0.083 & Keskil \\
\hline ETH185 & 0 & 0 & - & & 1 & 25 & 0.167 & Keskil, Moxsogol \\
\hline TGLA53 & 1 & 11 & 0.083 & Keskil & 1 & 17 & 0.083 & Keskil \\
\hline SPS115 & 0 & 0 & - & & 1 & 50 & 0.083 & Alii \\
\hline Average & & 3 & & & & 13 & & \\
\hline
\end{tabular}


Table 3: Average molecular coancestries

\begin{tabular}{|c|c|}
\hline Individual & Mean molecular coancestry \\
\hline Total population & 0.42 \\
\hline 23 bulls & 0.41 \\
\hline 17 bulls & 0.42 \\
\hline 6 bulls & 0.40 \\
\hline JA34 & 0.47 \\
\hline JA32 & 0.45 \\
\hline JA5 & 0.44 \\
\hline JA41 & 0.44 \\
\hline JA42 & 0.43 \\
\hline JA9 & 0.43 \\
\hline Alii & 0.42 \\
\hline JA38 & 0.42 \\
\hline JA 17 & 0.42 \\
\hline JA44 & 0.42 \\
\hline JA33 & 0.42 \\
\hline Moxsogol & 0.42 \\
\hline JA40 & 0.41 \\
\hline JA3 & 0.41 \\
\hline JA43 & 0.40 \\
\hline Sarial & 0.40 \\
\hline Radzu & 0.40 \\
\hline JA11 & 0.40 \\
\hline JA39 & 0.40 \\
\hline JA31 & 0.39 \\
\hline Erel & 0.38 \\
\hline JA16 & 0.38 \\
\hline Keskil & 0.34 \\
\hline
\end{tabular}

Values are calculated across pairwise comparisons between the individuals in the total population ( 60 individuals $=6$ cryo-bank bulls +17 contemporary bulls +37 contemporary cows) and the contemporary 37 cows, between the individuals in the total bull subpopulation ( 6 cryo-bank bulls +17 contemporary bulls) and the contemporary 37 cows, between the bulls (17 individuals) and the cows ( 37 individuals) from the Yakutian reference population, between the six Yakutian cryo-bank bulls and the cows (37 individuals), and between each Yakutian bull and the cows (37 individuals) separately

data provide a useful tool to estimate relatedness among individuals when pedigree data are unavailable. Moreover, the results clearly demonstrate the importance of $e x$ situ cryo-banking of genetic material in the conservation of rare domestic animal breeds.

\section{Relatedness}

Our microsatellite analysis suggests that five of the 15 pairwise relatedness comparisons for the Yakutian cryobank bulls exhibited coefficients of relatedness $(R)$ close to the theoretical expectations for half-sibs $(R=25 \%)$ and cousins $(R=12.5 \%)$. However, the two pairwise relatedness estimators identified different Yakutian bull-pairs as clear outliers compared to the simulated distribution of random individuals (Figure 1). Relatedness estimates for simulated unrelated pairs have a very wide distribution: the 95th percentiles $\left(r_{\mathrm{QG}}=0.242\right.$ and $\left.r_{\mathrm{W}}=0.252\right)$ are very near or above the theoretical expectation for half-sibs $(R$ $=0.25)$. This indicates that the 30 microsatellite markers used here are insufficient for an unequivocal separation of related and unrelated individuals.

The number and genetic variability of markers as well as population structure might affect the robustness of different methods in the calculation of relatedness between individuals. It has been also demonstrated that there is no single best-performing estimation method to distinguish between all possible types of relatedness [38-40]. In this study, $r_{\mathrm{W}}$ worked better for the simulated categories of related individuals that are important in solving relatedness questions among Yakutian cryo-bank bulls. The approach by [15] is robust for a small sample size and in the cases when the reference population includes unidentified relatives. These assumptions match closely the situation of the Yakutian population studied and therefore could explain the better performance of the estimator.

Thirty markers seems to be sufficient to identify PO's or FS's, but fails to separate HS's or more distant relatedness categories unequivocally. Additional simulations have demonstrated that a set of as many as 500 microsatellites would be needed for much more accurate estimates of $R$ with lower standard deviations (results not presented). Our results agree with previous suggestions that a large number of microsatellite loci are needed for unequivocal clarification of pedigrees [33]. Alternatively, using advanced SNP-microchips with thousands of SNP could provide a solution (e.g. [41]).

\section{Mitochondrial data}

MtDNA sequence analysis has shown that the Yakutian cryo-bank bulls do not share any mtDNA haplotype. Nucleotide substitutions accumulate approximately 5 to 10 times faster in mtDNA than in nuclear DNA [42] and cases of mtDNA mutation fixation within one generation have been described in Holstein cattle [43]. However, the smallest pairwise differences between haplotypes observed in Yakutian cryo-bank bulls were two nucleotides. As a result of heteroplasmy, the sons of a dam can have different mtDNA haplotypes. However, no heteroplasmy was detected in the present study. The mtDNA sequence analysis suggests that there are no full-sibs or maternal half-sibs among the Yakutian cryo-bank bulls. Although four Y chromosome-specific microsatellites (INRA124, INRA189, BM861 and BYM-1; see [44]) are monomorphic in the population [23], the mean related- 
ness based on the autosomal microsatellites shows that there are four potential half-sib pairs among the six Yakutian cryo-bank bulls.

\section{Allelic diversity and gene diversity}

The six Yakutian cryo-bank bulls appear to represent an important source of additional allelic variation and gene diversity for the Yakutian bull subpopulation as well as for the total Yakutian population. A high level of genetic diversity would determine the fitness of individuals and would affect the potential response of a population to immediate natural or artificial selection [45].

\section{Practical recommendations}

In a small population, misclassifying related individuals as unrelated (type II error) will result in underestimating relatedness within the population and, thus, represents a risk of increased inbreeding rate in subsequent generations. Therefore, we are more concerned about minimizing the occurrence of type II errors rather than the presence of type I errors, where unrelated individuals are identified as related. In the conservation program for the Yakutian cattle, we recommend that four Yakutian cryobank bull-pairs, Keskil:Moxsogol, Radzu:Sarial, Erel:Sarial and also Radzu:Erel, are treated as half-sibs or individuals otherwise having relatedness up to $25 \%$.

In an endangered population, choosing optimal individuals for mating and designing an appropriate mating scheme can help to monitor the genetic variation and the average relatedness among individuals. It has been shown that mating individuals with minimal average coancestries will maximize the population's genetic diversity in terms of expected heterozygosity $[46,47]$. In our study, 23 Yakutian bulls are candidate sires for the subsequent generation. However, as compared with the contemporary 17 bulls, the six Yakutian cryo-bank bulls show significantly lower average molecular coancestries with the cow population. Using the six cryo-bank semen in artificial insemination would help to control the rate of inbreeding in following generations.

The choice of the mating system is complicated because of the time scale of interest. From a short-term perspective, a simple breeding scheme could be suggested, whereby a population is subdivided into several groups and rotation mating among these groups is performed [48]. In the rotation mating scheme, breeding cows are from the same group as the sire, while breeding bulls are from a different group. Although this scheme will not reduce the degree of inbreeding in the long-run, a more even distribution of inbreeding among individuals would be achieved. Furthermore, it would guarantee that each line produces progeny that will be used for breeding in the next generation. On the basis of the pairwise relatedness among the six Yakutian cryo-bank bulls, we sug- gest to split them into three separate groups in the rotation mating, with Alii alone in another group, Keskil and Moxogol in one group, and Radzu, Erel and Sarial in a third group.

\section{Conclusions}

With the Yakutian cattle as an example, our results indicate that even a limited number of semen samples selected for the long-term cryo-banking can represent a considerable potential to maintain within-population genetic variability. Therefore, we recommend enrichment of the cryo-bank by adding semen of unrelated bulls with new genetic variability from the current living population. We have shown that when pedigree documentation is unavailable, even a limited number of molecular markers can help to make effective breeding mating schemes, though a larger set of markers would be desirable. We conclude that the present strategy with the help of molecular data can be applied to other animal species or even plants where the reduction of inbreeding and the preservation of genetic variation are important concerns.

\section{Additional material}

Additional file 1 Alignment of the variable sites in the $\mathbf{2 5 5} \mathrm{nt}$ fragment of the cattle mtDNA control region.

Additional file 2 Average relatedness estimates for pairwise comparisons among the six Yakutian cryo-bank bulls obtained using relatedness estimators $r_{\mathrm{W}}, r_{\mathrm{QG}}$ and $r_{\mathrm{K}}$

Additional file 3 Mean relatedness and their standard deviations of the two relatedness estimators $\left(r_{\mathrm{QG}}\right.$ and $\left.r_{\mathrm{W}}\right)$ for the four simulated relatedness categories.

Additional file 4 Results of the shared parentage analysis.

Competing interests

The authors declare that they have no competing interests.

\section{Authors' contributions}

IT designed the study, did the laboratory work, performed the data analysis and drafted the manuscript. MT contributed to the data analysis and the draft writing. MHL contributed to the draft writing and revised the manuscript critically. RP and ZY contributed to the sample collection and the manuscript writing and provided important expertise on Yakutian cattle. JK planned and coordinated the whole study, and contributed to the manuscript writing. All the authors read and approved the final manuscript.

\section{Acknowledgements}

We are indebted to A. Virta for her technical assistance in microsatellite genotyping and mtDNA sequencing, to $V$. Ahola for her help in bioinformatics and to M. Toro for his assistance in coancestry analyses. This work was performed as a part of the Russian in Flux-research programme of the Academy of Finland and was financially supported by the Academy of Finland. The office space provided by ILRI in Nairobi, Kenya for I. Tapio at the final stage of the study is acknowledged.

\section{Author Details}

1Biotechnology and Food Research, MTT Agrifood Research Finland, Jokioinen, Fl-31600 Finland and ${ }^{2}$ Yakutian Research Institute of Agriculture, 677002

Yakutsk, Sakha, Russia

Received: 29 April 2010 Accepted: 13 July 2010

Published: 13 July 2010 


\section{References}

1. Granberg L, Soini K, Kantanen J: Sakha Ynaga-cattle of the Yakuts Helsinki: Finnish Academy of Science and Letters; 2009.

2. Li MH, Nogovitsina E, Ivanova Z, Erhardt G, Vilkki J, Popov R, Ammosov I, Kiselyova T, Kantanen J: Genetic contribution of indigenous Yakutian Cattle to two hybrid populations, revealed by microsatellite variation. Asian-Aust J Anim Sci 2005, 18:613-619.

3. Li MH, Tapio I, Vilkki J, Ivanova Z, Kiselyova T, Marzanov N, Ćinkulov M, Stojanovic S, Ammosov I, Popov R, Kantanen J: The genetic structure of cattle populations (Bos taurus) in northern Eurasia and the neighbouring Near Eastern regions: implications for breeding strategies and conservation. Mol Ecol 2007, 16:3839-3853.

4. Scherf BD: World Watch List for Domestic Animal Diversity 3rd edition. Rome: Food and Agriculture Organization of the United Nations; 2000

5. Bennewitz J, Kantanen J, Tapio I, Li M-H, Kalm E, Vilkki J, Ammosov I, Ivanova Z, Kiselyova T, Popov R, Meuwissen THE: Estimation of breed contributions to present and future genetic diversity of 44 North Eurasian cattle breeds using core set diversity measures. Genet Sel Evol 2006, 38:201-220

6. Sigurdsson A, Jonmundsson JV: Inbreeding and its impact in the closed population of Icelandic dairy cattle. Acta Agric Scand A 1995, 45:11-16.

7. Frankham R: Stress and adaptation in conservation genetics. J Evol Biol 2005, 18:750-755.

8. Garner A, Rachlow JL, Hicks J: Patterns of genetic diversity and its loss in mammalian populations. Conserv Biol 2005, 19:1215-1221.

9. Meuwissen T: Operation of conservation schemes. In Utilisation and conservation of farm animal genetic resources Edited by: Oldenbroek K. Wageningen: Academic Publishers; 2007:167-193.

10. Slate J, Marshall T, Pemberton J: A retrospective assessment of the accuracy of the paternity inference program CERVUS. Mol Ecol 2000, 9:801-808.

11. Li MH, Välimäki K, Piha M, Pakkala T, Merilä J: Extrapair paternity and maternity in the three-toed woodpecker, Picoides tridactylus: insights from microsatellite-based parentage analysis. PLOS ONE 2009, 4:e7895.

12. Li MH, Merilä J: Sex-specific population structure, natural selection, and linkage disequilibrium in a wild bird population as revealed by genome-wide microsatellite analyses. BMC Evol Biol 2010, 10:66

13. Queller DC, Goodnight KF: Estimating relatedness using genetic markers. Evolution 1989, 43:258-275

14. Lynch M, Ritland K: Estimation of pairwise relatedness with molecular markers. Genetics 1999, 152:1753-1766.

15. Wang J: An estimator for pairwise relatedness using molecular markers. Genetics 2002, 160:1203-1215

16. Jones AG, Arden WR: Methods of parentage analysis in natural populations. Mol Ecol 2003, 12:2511-2523

17. Kalinowski ST, Wagner AP, Taper ML: ML-Relate: a computer program for maximum likelihood estimation of relatedness and relationship. $\mathrm{Mol}$ Ecol Notes 2006, 6:576-579.

18. Epstein MP, Duren WL, Boehnke M: Improved inference of relationships for pairs of individuals. Am J Hum Genet 2000, 67:1219-1231.

19. Butler K, Field C, Herbinger CM, Smith BR: Accuracy, efficiency and robustness of four algorithms allowing full sibship reconstruction from DNA marker data. Mol Ecol 2004, 13:1589-1600.

20. Toro M, Barragan C, Ovilo C, Rodriganez J, Rodriguez C, Silio L: Estimation of coancestry in Iberian pigs using molecular markers. Conserv Genet 2002, 3:309-320.

21. Zadworny D, Kuhnlein U: The identification of the kappa-casein genotype in Holstein dairy cattle using the polymerase chain reaction. Theor App/ Genet 1990, 80:631-634

22. Cymbron T, Loftus RT, Malheiro MI, Bradley DG: Mitochondrial sequence variation suggests an African influence in Portuguese cattle. Proc R SOC London B 1999, 266:597-603.

23. Kantanen J, Edwards CJ, Bradley DG, Viinalass H, Thessler S, Ivanova Z, Kiselyova T, Cinkulov M, Popov R, Stojanović S, Ammosov I, Vilkki J: Maternal and paternal genealogy of Eurasian taurine cattle (Bos taurus). Heredity 2009, 103:404-415.

24. Thompson JD, Gibson TJ, Plewniak F, Jeanmougin F, Higgins DC: The ClustalX windows interface: flexible strategies for multiple sequence alignment aided by quality analysis tools. Nucleic Acids Res 1997 25:4876-4882
25. Kumar S, Tamura K, Nei M: MEGA 3: integrated software for molecular evolutionary genetics analysis and sequence alignment. Brief Bioinform 2004, 5:150-163.

26. Park S: Microsatellite TOOLKIT: Excel Add-in Tool Package for Microsatellite Data. Genetics Department, Trinity College, Dublin; 2001

27. Guo SW, Thompson EA: Performing the exact test of Hardy-Weinberg proportions for multiple alleles. Biometrics 1992, 48:361-372.

28. Rousset F, Raymond M: Testing heterozygote excess and deficiency. Genetics 1995, 140:1413-1419.

29. Raymond M, Rousset F: GENEPOP (version 1.2): population genetics software for exact tests and ecumenicism. J Hered 1995, 86:248-249.

30. Hardy OJ, Vekemans X: SPAGeDi: a versatile computer program to analyse spatial genetic structure at the individual or population levels. Mol Ecol Notes 2002, 2:618-620.

31. Coombs JA, Letcher BH, Nislow KH: PEDAGOG: Software for simulating eco-evolutionary population dynamics. 2008 [https:// bcrc.bio.umass.edu/pedigreesoftware/

32. Emery AM, Wilson IJ, Craig S, Boyle PR, Noble LR: Assignment of paternity groups without access to parental genotypes: multiple mating and developmental plasticity in squid. Mol Ecol 2001, 10:1265-1278.

33. Malécot G: Les Mathématiques de l'Hérédité. Masson et Cie, Paris, France 1948

34. Fernandez J, Toro MA: A new method to estimate relatedness from molecular markers. Mol Ecol 2006, 15:1657-1667.

35. Troy CS, MacHugh DE, Bailey JF, Magee DA, Loftus RT, Cunningham P Chamberlain AT, Sykes BC, Bradley DG: Genetic evidence for NearEastern origins of European cattle. Nature 2001, 410:1088-1091.

36. Mannen H, Kohno M, Nagata Y, Tsuji S, Bradley DG, Yeo JS, Nyamsamba D, Zagdsuren Y, Yokohama M, Nomura K, Amano T: Independent mitochondrial origin and historical genetic differentiation in North Eastern Asian cattle. Mol Phylogenet Evol 2004, 32:539-544.

37. Kantanen J, Olsaker I, Holm L-E, Lien S, Vilkki J, Brusgaard K, Eythorsdottir E, Danell B, Adalsteinsson S: Genetic diversity and population structure of 20 North European cattle breeds. J Hered 2000, 91:446-457.

38. Van de Casteele T, Galbusera P, Matthysen E: A comparison of microsatellite-based pairwise relatedness estimators. Mol Ecol 2001 10:1539-1549.

39. Russello MA, Amato G: Ex situ population management in the absence of pedigree information. Mol ECO/ 2004, 13:2829-2840.

40. Oliehoek PA, Windig JJ, van Alendonk JAM, Bijma P: Estimating relatedness between individuals in general populations with a focus on their use in conservation programs. Genetics 2006, 173:483-496.

41. Langlois B: Optimising the choice of molecular markers for inbreeding analyses. In Proceedings of the 8th World congress on genetics applied to livestock production: 13-18 August 2006 Belo Horizonte; 2006:08-02.

42. Brown WM, Prager EM, Wang A, Wilson AC: Mitochondrial DNA sequence of primates: tempo and mode of evolution. J Mol Biol 1982, 18:225-239.

43. Koehler CM, Lindberg GL, Brown DR, Beitz DC, Freeman AE, Mayfield JE, Myers AM: Replacement of bovine mitochondrial DNA by sequence variant within one generation. Genetics 1991, 129:247-255

44. Li MH, Zerabruk M, Vangen O, Olsaker I, Kantanen J: Reduced genetic structure of north Ethiopian cattle revealed by Y-chromosome analysis. Heredity 2007, 98:214-221.

45. James J: The founder effect and response to artificial selection. Genet Res 1971, 12:249-266.

46. Lacy R: Clarification of genetic terms and their use in the management of captive populations. Zoo Biol 1995, 14:565-578.

47. Caballero A, Toro MA: Interrelations between effective population size and other pedigree tools for the management of conserved populations. Genet Res 2000, 75:331-343.

48. Verrier $\mathrm{E}$, Rochambeau $\mathrm{H}$ : Problems of inbreeding in small populations and management of rare breeds. Data collection, conservation and use of farm animal genetic resources European workshop 1992:2-17.

doi: $10.1186 / 1297-9686-42-28$

Cite this article as: Tapio et al., Estimation of relatedness among non-pedigreed Yakutian cryo-bank bulls using molecular data: implications for conservation and breed management Genetics Selection Evolution 2010, 42:28 